melanoma cell line A101D to analyze the response to three conditions: methionine depletion, methionine replacement with homocysteine, and cysteine depletion in vitro. We measured proliferation/ viability, gene expression patterns, and glutathione levels. We also assessed ferroptotic versus apoptotic cell death. We then used a normal human fibroblast cell line to compare responses. RESULTS/ ANTICIPATED RESULTS: The transcription factors ATF4 and NRF2 were activated by all three tested conditions in melanoma cells. Glutathione levels were decreased by $\sim 40 \%$ in cells grown without methionine, and by $95 \%$ in cells grown without cysteine. Lipid peroxidation was increased in cells grown without cysteine, but not in cells grown without methionine. Inhibiting ferroptotic cell death partially rescued proliferation in cysteine-depleted but not in methionine-depleted cells. Almost $70 \%$ of cells grown in methioninedepleted media stained positive for Annexin V, an indicator of apoptosis, compared to only $20 \%$ of cells grown in cysteine-depleted media. In normal cells, ferrostatin recued proliferation/viability to $86 \%$ of control levels in cysteine-depleted cells. Ferrostatin did not affect methionine-depleted normal cells. DISCUSSION/ SIGNIFICANCE OF FINDINGS: These results indicate that methionine depletion leads to apoptosis, while cysteine depletion leads to ferroptosis. We found overlapping pathways activated by methionine and cysteine depletion at the gene expression levels, but divergences in cell death pathways ultimately activated.

32329

\section{A novel mouse model of Ataxia Telangiectasia for testing small molecule readthrough compounds}

Paul Mathews

The Lundquist Institute for Biomedical Innovation at Harbor-UCLA Medical Center

ABSTRACT IMPACT: Small molecule readthrough compounds are a promising therapeutic with the potential to overcome nonsense mutations thereby enabling the production of functional ATM protein in patients with Ataxia Telangiectasia OBJECTIVES/GOALS: To generate a novel mouse model of Ataxia-Telangiectasia for testing small molecule readthrough compounds that both expresses a clinically relevant nonsense mutation and recapitulates the major symptoms of the disease, including a progressive loss of motor coordination not previously observed in prior A-T animal models. METHODS/STUDY POPULATION: Using a double-hit strategy to increase genotoxic stress, we generated a novel A-T mouse model that expresses a clinically relevant $(\mathrm{c} .103 \mathrm{C}>\mathrm{T})$ mutation in the Atm gene and a knockout of the functionally related Aptx gene. We then characterized the mouse across multiple domains related to the various symptoms related to the disease. This includes examination of survivability, immunologic function, cancer prevalence, and motor behavior and its associated cerebellar dysfunction and atrophy. Lastly, we tested the ability of small molecule readthrough compounds to enable production of ATM from tissue explants extracted from these ATM deficient mice. RESULTS/ANTICIPATED RESULTS: The double mutant mice display reduced survivability compared to control mice ( $53 \%$ vs. $97 \%$; $<<0.0001$ ), dying at a clinically relevant rate of about $30 \%$ from thymomas. At postnatal day 400 (P400), only AtmR35X/R35X; Aptx-/- mice, and none of the controls expressing at least one wildtype Atm or Aptx gene develop a motor behavioral deficits that are associate with reduced Purkinje neuron diameter $(8.0 \pm 0.4 \mu \mathrm{m}$ vs. $9.92 \pm 0.5 ; \mathrm{p}<0.01)$ and density $(4.3$ \pm 0.2 vs. $6.0 \pm 0.3$ per $100 \mu \mathrm{m} ; \mathrm{p}<0.05)$ as well as cerebellar atrophy (cerebellum/forebrain area $0.26 \pm 0.01$ vs. $0.31 \pm 0.01 ; \mathrm{p}<0.001$ ). ATM deficient mice also display disrupted thymocyte development and metabolic function. When exposed to small molecular readthrough compounds, greater than $50 \%$ of the ATM protein is restored. DISCUSSION/SIGNIFICANCE OF FINDINGS: We have created a novel, clinically relevant A-T mouse model that develops a severe ataxia associated with changes in cerebellar function and atrophy as well as demonstrate the potential of SMRT compounds as an A-T therapeutic.

33886

\section{Deregulation of the LANCL2 Pathway in Alzheimer's Disease \\ Emily Birnbaum, Maria Figueiredo-Pereira, Patrica Rockwell, Claire Henchcliffe and Peter Serrano \\ ${ }^{1}$ CUNY Graduate Center, Hunter College, Weill Cornell Medicine, ${ }^{2}$ Hunter College and ${ }^{3}$ Weill Cornell Medicine, UC Irvine School of Medicine}

ABSTRACT IMPACT: My work focuses on understanding the role of an immunomodulatory pathway in Alzheimer's disease as a potential therapeutic target. OBJECTIVES/GOALS: My long-term goal is to develop effective drugs to halt the progression of Alzheimer's disease (AD). The overall objective of this translational work is to study the deregulation of the Lanthionine Synthetase C-like 2 (LANCL2) immunomodulatory pathway in $\mathrm{AD}$. I hypothesize that stimulation of the LANCL2 pathway will inhibit progression of AD. METHODS/ STUDY POPULATION: These studies use a transgenic rat model of $\mathrm{AD}$, and a LANCL2-binding drug, BT-11, that crosses the BBB. TgAD Fisher 344 rats express human APP with the Swedish mutation and human presenilin- 1 with the ? exon 9 mutation. Tg-AD rats develop age-dependent AD-like pathology. Rodent chow containing BT-11 $(10 \mathrm{mg} / \mathrm{Kg} \mathrm{bw})$ was administered orally from 5 months of age (pre-pathology) to 11 months of age. Eight groups of rats (8 rats/group) were included: WT and Tg-AD x 2 sexes x 2 conditions (BT-11 treated and untreated). Rats are analyzed for: (1) Spatial learning and memory with an active place avoidance test. (2) AD pathology including amyloid plaques and activated microglia, with hippocampal immunohistochemistry. (3) LANCL2-signaling and other AD relevant pathways, with hippocampaI western blot and RNAseq analyses. RESULTS/ ANTICIPATED RESULTS: I predict that BT-11 will prevent and/or mitigate some aspects of $\mathrm{AD}$ pathology displayed by the $\mathrm{AD}$ transgenic rats (Tg-AD). Tg-AD rats treated with $\mathrm{BT}-11$ should perform better in the spatial learning and memory tasks, than the untreated $\mathrm{Tg}-\mathrm{AD}$ rats. I also expect that the immunohistochemical analysis will reveal reduced pathological hallmarks, including amyloid plaques and reactive microglia, in hippocampal tissue of BT-11 treated versus untreated Tg-AD rats. Through western blot and RNAseq analyses, I will establish how BT-11 treatment affects the LANCL2 signaling cascade as well as other AD relevant pathways, in treated and untreated rats from both sexes and both genotypes. DISCUSSION/SIGNIFICANCE OF FINDINGS: My proposed translational research will address the potential of targeting the LANCL2 pathway to improve AD treatment. Discovering the effects of stimulating the LANCL2 pathway on AD pathology is a novel approach in $\mathrm{AD}$ drug development. I expect that my studies with BT-11 will positively influence therapeutic outcomes of this devastating condition. 